\title{
Chronic depression of hypothalamic paraventricular neuronal activity produces sustained hypotension in hypertensive rats
}

\author{
Vera Geraldes ${ }^{1}$, Nataniel Gonçalves-Rosa ${ }^{1}$, Beihui Liu ${ }^{2}$, Julian F. R. Paton ${ }^{2}$ and Isabel Rocha ${ }^{1,3}$ \\ ${ }^{1}$ Cardiovascular Autonomic Function Laboratory, Faculty of Medicine and Instituto de Medicina Molecular and ${ }^{3}$ Centro de Cardiologia, University of \\ Lisbon, Portugal \\ ${ }^{2}$ School of Physiology \& Pharmacology, Bristol Heart Institute, Medical Sciences Building, University of Bristol, Bristol BS8 1TD, UK
}

\section{New Findings}

- What is the central question of this study?

Will a chronic reduction of neuronal excitability within the paraventricular nucleus of the hypothalamus reduce arterial blood pressure and sympathetic activity in the long term in an animal model of neurogenic hypertension?

- What is the main finding and its importance?

We show, for the first time, that overexpression of an inwardly rectifying potassium channel in the paraventricular nucleus provided a long-term ( $>60$ days) antihypertensive response in conscious spontaneously hypertensive rats that was associated with a reduction in neurohumorally mediated vasoconstriction, enhanced baroreflex sensitivity and reduced peripheral chemosensitivity; no such response was observed in normotensive rats. Our results support the paraventricular nucleus as a therapeutic target for the chronic control of blood pressure in neurogenic hypertension.

Changes in the sympathetic nervous system are responsible for the initiation, development and maintenance of hypertension. An important central sympathoexcitatory region is the paraventricular nucleus (PVN) of the hypothalamus, which may become more active in hypertensive conditions, as shown in acute studies previously. Our objective was to depress PVN neuronal activity chronically by the overexpression of an inwardly rectifying potassium channel (hKir2.1), while evaluating the consequences on blood pressure (BP) and its reflex regulation. In spontaneously hypertensive rats (SHRs) and Wistar rats (WKY) lentiviral vectors (LVVhKir2.1; LV-TREtight-Kir-cIRES-GFP5 $4 \times 10^{9}$ IU and LV-Syn-Eff-G4BS-Syn-Tetoff $6.2 \times 10^{9}$ IU in a ratio 1:4) were stereotaxically microinjected bilaterally into the PVN. Sham-treated SHRs and WKY received bilateral PVN microinjections of LVV-eGFP (LV-Syn-Eff-G4BS-Syn-Tetoff $6.2 \times 10^{9} \mathrm{IU}$ and LV-TREtight-GFP $5.7 \times 10^{9} \mathrm{IU}$ in a ratio $\left.1: 4\right)$. Blood pressure was monitored continuously by radio-telemetry and evaluated over 75 days. Baroreflex gain was evaluated using phenylephrine $\left(25 \mu \mathrm{g} \mathrm{ml}^{-1}\right.$, I.V.), whereas lobeline $\left(25 \mu \mathrm{g} \mathrm{ml}^{-1}\right.$, I.v. $)$ was used to stimulate peripheral chemoreceptors. In SHRs but not normotensive WKY rats, LVV-hKir2.1 expression in the PVN produced time-dependent and significant decreases in systolic (from $158 \pm 3$ to $132 \pm 6 \mathrm{mmHg} ; P<0.05$ ) and diastolic BP (from $135 \pm 4$ to $113 \pm 5 \mathrm{mmHg} ; P<0.05$ ). The systolic BP low-frequency band was reduced (from $0.79 \pm 0.13$ to $0.42 \pm 0.09 \mathrm{mmHg}^{2} ; P<0.05$ ), suggesting reduced sympathetic vasomotor tone. Baroreflex gain was increased and peripheral chemoreflex depressed after PVN microinjection of LVV-hKir2.1. We conclude that the PVN plays a major role in long-term control of BP and sympathetic nervous system activity in SHRs. 
This is associated with reductions in both peripheral chemosensitivity and respiratory-induced sympathetic modulation and an improvement in baroreflex sensitivity. Our results support the $\mathrm{PVN}$ as a powerful site to control BP in neurogenic hypertension.

(Received 25 June 2013; accepted after revision 15 October 2013; first published online 18 October 2013)

Corresponding author I. Rocha: Instituto de Fisiologia, Faculdade de Medicina de Lisboa, Av Prof Egas Moniz, 1649-028

Lisbon, Portugal. Email: isabelrocha@fm.ul.pt

\section{Introduction}

Essential arterial hypertension has now reached pandemic proportions, with an estimated one billion sufferers worldwide. The pathogenesis of essential arterial hypertension is multifactorial and not completely understood, but there is clear evidence that chronic elevation of sympathetic nervous system activity is a major contributor to the onset, development and maintenance of the hypertensive state (Grassi, 2004b; Guyenet, 2006; Fisher \& Paton, 2012). In fact, the increase of sympathetic outflow to the heart results in increased cardiac output and neurally mediated vasoconstriction, leading to elevated blood pressure values (Schlaich et al. 2012). In 'white coat' and borderline hypertensive patients, sympathetic nerve activity to the arterioles supplying skeletal muscle is raised in comparison to healthy individuals (Grassi, 2004a; Smith et al. 2004). Excessive sympathetic activity may contribute to hypertrophy of vascular smooth muscle and cardiac muscle, brain hypoperfusion and inflammation, and becomes a major target to control in neurogenic hypertension (Zubcevic et al. 2011).

The evaluation of sympathetic activity can be achieved indirectly by applying mathematical tools such fast Fourier transform to blood pressure signals (Task Force of the European Society of Cardiology and the North American Society of Pacing and Electrophysiology, 1996). A power spectrum is generated, where the low frequencies (LFs) represent predominantly sympathetic activity and high frequencies (HFs) are related to parasympathetic tonus and respiration (Radaelli et al. 1994; Task Force of the European Society of Cardiology and the North American Society of Pacing and Electrophysiology, 1996; Furlan et al. 2000). These mathematical results have been addressed in several studies, which have suggested that sympathetic activity is a critical determinant of blood pressure fluctuations in a frequency range which is slower than the rate of respiration (Japundzic et al. 1990; Cerutti et al. 1991; Malliani et al. 1991).

Located in the hypothalamus, the paraventricular nucleus (PVN) is a major sympathoexcitatory area that becomes more active in conditions of hypertension, such as in the spontaneously hypertensive rat (SHR) model (Allen, 2002). Some authors have referred to this region as a command nucleus providing feedforward excitatory synaptic drives to co-ordinate lower brainstem cardiovascular and respiratory motor activity (Dampney et al. 2005). Activation of the PVN promotes an increase in sympathetic output and a pressor effect mediated via direct and indirect projections (via the rostral ventrolateral medulla) to the spinal cord (Caverson et al. 1984; Shafton et al. 1998; Pyner \& Coote, 2000; Hardy, 2001).

Both electrical stimulation and chemical manipulation of PVN neurons with bicuculline $\left(\mathrm{a} \mathrm{GABA}_{\mathrm{A}}\right.$ receptor antagonist) or glutamate elevated sympathetic nerve activity and caused hypertension in anaesthetized and conscious rats (Kannan et al. 1989; Zhang et al. 2002). In contrast, acute inhibition of the PVN with GABA or muscimol reduces the blood pressure and sympathetic nerve activity in SHRs (Allen, 2002). Lesions of the PVN or transection of the brain caudal to the hypothalamus promotes a decrease in blood pressure in SHRs but not in Wistar-Kyoto (WKY) rats (Yamori \& Okamoto, 1969; Goto et al. 1981; Ciriello et al. 1984; Herzig et al. 1991; Takeda et al. 1991).

Long-term manipulation of neurone excitability can be performed by expressing a human inwardly rectifying potassium channel (hKir2.1) under the control of a selective neuronal promoter, such as synapsin (Duale et al. 2005 , 2007). Inwardly rectifying potassium channels, such as Kir2.1, are endogenously expressed in rat brain and have recently been overexpressed as a means to reduce neuronal membrane excitability (Yu et al. 2004; Duale et al. 2007; Mizuno et al. 2007; Okada \& Matsuda, 2008; Yoon et al. 2008; Howorth et al. 2009). Their long-term expression can be achieved by the use of lentiviral vectors (LVVs) derived from human immunodeficiency virus (Coleman et al. 2003). Therefore, using a LVV to overexpress hKir2.1 channels within the PVN, we sought to determine the long-term influence of this nucleus on the control of blood pressure, heart rate, sympathetic activity and respiration in SHRs, as well as homeostatic reflex control mechanisms.

\section{Methods}

All the experimental procedures were in accordance with the European and Portuguese Law on animal welfare and had the approval of the ethics committee of the Faculty of Medicine, University of Lisbon, Portugal. Male WistarKyoto rats $(n=15)$ and SHRs $(n=15)$ were used, aged 12 weeks and weighing $363 \pm 8 \mathrm{~g}$. Animals, synchronized to a $12 \mathrm{~h}-12 \mathrm{~h}$ light-dark cycle (light on at $07.00 \mathrm{~h}$ and light off at $19.00 \mathrm{~h}$ ), were housed individually and allowed to freely move in standard plastic cages. Food and water were available ad libitum. 


\section{Viral vector construction and validation}

Lentiviral vector construction was based on previous studies (Waki et al. 2003; Duale et al. 2007). Briefly, LVVeGFP, used for the sham-treated group, was a mix of LVTREtight-GFP $5.7 \times 10^{9}$ IU and LV-Syn-Eff-G4BS-SynTetoff $6.2 \times 10^{9}$ IU in a ratio $1: 4$. This binary system expresses enhanced green fluorescent protein (eGFP). The LVV-hKir2.1 is a mix of LV-TREtight-Kir-cIRES-GFP $5.4 \times 10^{9}$ IU and LV-Syn-Eff-G4BS-Syn-Tetoff $6.2 \times 10^{9}$ IU in a ratio 1:4, which expresses eGFP and expresses human inwardly rectifying potassium channels (hKir2.1) in neurones. Validation of transduction efficacy and transgene expression was assessed as described previously by Duale et al. (2007) and included mRNA expression, immunocytochemical and electrophysiological data.

\section{Microinjection sites}

Initially, we fine tuned our stereotaxic co-ordinates for bilateral PVN microinjections in five SHRs and five WKY rats anaesthetized with sodium pentobarbitone (60 $\mathrm{mg} \mathrm{kg}^{-1}$, I.P., Hikma Pharmaceuticals, London, UK). Bilateral microinjections $(0.05 \mu \mathrm{l})$ of LVV-eGFP were performed. Using fluorescence microscopy and histological reconstruction, we determined the correct coordinates for PVN injections and the amount of LVV-eGFP needed to limit transduction to the confines of the PVN.

\section{Surgery}

Spontaneoulsy hypertensive rats were divided into two groups according to the microinjection content, i.e. LVV-hKir2.1 $(n=8)$ and LVV-eGFP $(n=7)$. A control group of WKY rats, with matching age, sex and number of individuals, underwent the same surgical and experimental protocol.

Implantation of telemetry probes. Rats were implanted with radio-telemetry probes (DSI, St. Paul, Minnesota, $\mathrm{MN}$, USA) in the abdominal aorta under general anaesthesia (sodium pentobarbitone, $60 \mathrm{mg} \mathrm{kg}^{-1}$, I.P., Hikma Pharmaceuticals). Animals were allowed to recover for 15 days. Similar anaesthetic and surgical protocols were applied to WKY rats $(n=15)$.

Bilateral microinjection in the PVN. Two weeks after the probes were implanted, SHRs $(n=8)$ and WKY rats $(n=8)$ under general anaesthesia (sodium pentobarbitone, $60 \mathrm{mg} \mathrm{kg}^{-1}$, I.P., Hikma Pharmaceuticals) were placed in a stereotactic frame (Kopf Instruments, Tujunga, CA, USA), and a craniotomy was performed using our previously determined co-ordinates for LVVhKir2.1 microinjections $(0.05 \mu \mathrm{l})$ into the PVN (Bregma, $-1.6 \mathrm{~mm}$; Lateral, $\pm 1.41 \mathrm{~mm}$; Deep, $7.4 \mathrm{~mm}$; pipette angle, 10 deg to bregma; Paxinos \& Watson, 1986). Sham- treated rats were microinjected in the same region with LVV-eGFP (SHRs, $n=7$; and WKY rats, $n=7$ ). All microinjections were performed bilaterally. Animals of all groups were allowed to recover and monitored by telemetry for 60 days. Heart rate (HR) and blood pressure [BP; systolic (SBP), diastolic and mean] were recorded continuously.

\section{Metabolic evaluation}

Rats were housed for $24 \mathrm{~h}$ in metabolic cages to evaluate body weight, intake of food and fluid and production of urine and faeces. Measurements were performed before and 59 days after each microinjection.

\section{Cardiorespiratory reflex evaluation}

At 60 days, animals were anaesthetized (sodium pentobarbitone, $60 \mathrm{mg} \mathrm{kg}^{-1}$, I.P., Hikma Pharmaceuticals). The trachea was cannulated below the larynx to record tracheal pressure. The femoral and carotid arteries and femoral vein were cannulated. Rectal temperature was maintained at $38 \pm 1^{\circ} \mathrm{C}$ by a servocontrolled heating blanket. The ECG was recorded with the use of needle electrodes inserted into the limbs, and HR was derived from the ECG. Baroreceptor and peripheral chemoreceptor reflexes were activated twice, with an interval of $5 \mathrm{~min}$ between stimuli. Baroreceptor reflex was stimulated by phenylephrine $\left(0.2 \mathrm{ml}, 25 \mu \mathrm{g} \mathrm{ml}^{-1}\right.$ I.V.; Sigma Aldrich). Peripheral chemoreceptor reflex was stimulated with lobeline $\left(0.2 \mathrm{ml}, 25 \mu \mathrm{g} \mathrm{ml}^{-1}\right.$, Sigma Aldrich) injected retrogradely into the bifurcation of the common carotid artery. Heart rate, BP (systolic, diastolic and mean) and respiratory rate (RespR) were recorded continuously thought the experiment.

\section{Histology and immunochemistry}

Animals were terminally anaesthetized and immediately perfused transcardially with $\mathrm{PBS}(0.1 \mathrm{M}$; pH 7.4) followed by $4 \%$ paraformaldehyde $(0.1 \mathrm{M}$; $\mathrm{pH} 7.4)$. The brain was removed and placed for $48 \mathrm{~h}$ in $15 \%(\mathrm{w} / \mathrm{v})$ sucrose solution. Coronal sections $(18 \mu \mathrm{m}$ thick) were cut on a microtome and mounted on slides. The pipette tip location and the microinjection diffusion in the PVN were examined and documented. The microinjected contents (LVV-hKir2.1 or LVV-eGFP) containing eGFP allowed an estimation of virus dispersion. The eGFPlabelled fluorescent regions were identified using an epifluorescence microscope and plotted on standardized sections from the atlas of Paxinos \& Watson (1986).

\section{Western blot analysis}

The expression of hKir2.1 in the PVN was analysed by Western blot 60 days after the microinjection of 
LVV-hKir2.1 $(n=8)$ or LVV-eGFP in SHRs $(n=7)$. The $\mathrm{PVN}$ was dissected from both groups and homogenized by sonication in ice-cold RIPA buffer (Sigma, St. Louis, MO, USA) supplemented with a cocktail of protease inhibitors (complete mini; Roche). Proteins were extracted from the homogenates by centrifugation at $5000 \mathrm{~g}$ for $10 \mathrm{~min}$ at $4{ }^{\circ} \mathrm{C}$, and protein concentration was determined with a Bio-Rad DC Protein Assay kit. Proteins were resolved by electrophoresis on a $10 \%$ Tris-glycine SDS-PAGE gel and transferred to a polyvinylidene fluoride membrane (Millipore, Bedford, MA, USA). Membranes were blocked with 5\% milk in Tween/Tris-buffered saline and incubated overnight at $4{ }^{\circ} \mathrm{C}$ with rabbit anti-hKir2.1 polyclonal antibody (Abcam, Cambridge, UK). After washing, membranes were incubated for $1 \mathrm{~h}$ at room temperature with HRP-conjugated goat anti-rabbit antibody (Bio-Rad, Hercules, CA, USA), and immunoreactive proteins were detected by Immobilon Western Chemiluminescent HRP Substrate (Millipore, Bedford, MA, USA) and visualized using Curix 60 (AGFA, Greenville, SC, USA). Membranes were stripped with $0.1 \mathrm{M}$ glycine $(\mathrm{pH} 2.2)$ and reprobed with the $\alpha$-tubulin antibody (Santa Cruz Biotechnology, Dallas, TX, USA) for loading control.

\section{Data acquisition and analysis}

Telemetric data were acquired at $1 \mathrm{kHz}$ and analysed with suitable software (LabChart6, Powerlab; ADInstruments, Oxford, UK). Mean values of HR, BP (systolic, diastolic and mean) and RespR were extracted.

Baroreceptor and chemoreceptor reflex. The baroreceptor reflex gain (BRG) was quantified by calculating $\Delta \mathrm{HR} / \triangle \mathrm{BP}$ (in beats per minute per millimetre of mercury). Chemoreceptor (ChR) reflex was calculated through the RespR derived from the tracheal pressure before and after stimulation with lobeline, as follows: $\Delta \mathrm{ChR}=\mathrm{RespR}_{\text {lobeline }}-\mathrm{RespR}_{\text {basal }}$. Blood pressure and HR were also evaluated.

Analysis of BP and HR variability. Systolic BP and R$\mathrm{R}$ interval data were analysed (period of $3 \mathrm{~min}$ ) in the frequency domain (Fast Fourier Transform), using the in-house software Fisiosinal (Tavares, 2011), to evaluate sympathetic (LF band, $0.15-0.6 \mathrm{~Hz}$ of systolic BP) and parasympathetic activity ( $\mathrm{HF}$ band, $0.6-2.0 \mathrm{~Hz}$ of $\mathrm{HR}$ ) over time (Task Force of the European Society of Cardiology and the North American Society of Pacing and Electrophysiology, 1996; Marques-Neves et al. 2004).

Circadian light/dark heart rate and blood pressure profile. Mean BP and HR values were calculated using the continuous telemetric data and compared between light (07.00-19.00 h) and dark phases (19.00-07.00 h).

\section{Statistical analysis}

Comparisons were performed between groups for the same period and within the same group, before and after the microinjections. For the statistical analysis, Student's paired $t$ test and ANOVA for comparisons between groups were used. All data were expressed as means \pm SEM and passed the normality test. Significance was taken as $P<0.05$.

\section{Results}

Effect of microinjection of LVV-hKir2.1 or LVV-eGFP on $24 \mathrm{~h}$ mean values of blood pressure, heart rate and respiration

Basal BP values (recorded before microinjections) in conscious SHRs $(n=15)$ were $158 \pm 3 \mathrm{mmHg}$ for systolic $\mathrm{BP}, 135 \pm 4 \mathrm{mmHg}$ for diastolic $\mathrm{BP}$ and $142 \pm 3 \mathrm{mmHg}$ for mean BP, and were significantly higher than the values for WKY rats $(119 \pm 3,91 \pm 2$ and $101 \pm 1 \mathrm{mmHg}$, respectively; $n=15 ; P<0.0001)$. The SHRs showed a higher baseline respiratory rate than WKY rats $(77 \pm 5$ versus $61 \pm 4$ breaths $\min ^{-1}$, respectively; $\left.P<0.05\right)$ as well as a lower HR $\left(311 \pm 5\right.$ and $367 \pm 9$ beats $\mathrm{min}^{-1}$; $P<0.0001$ ).

Thirty days after LVV-hKir2.1 microinjection, a significant BP decrease $(P<0.05)$ was first observed, but in order to evaluate its persistence, animals were monitored for a further 30 days. On the 60th day after lentiviral microinjection, values for SHRs for systolic, diastolic and mean BP were $132 \pm 6,113 \pm 5$ and $120 \pm 5 \mathrm{mmHg}$, respectively, corresponding to a decrease in pressure of 26, 22 and $22 \mathrm{mmHg}$, respectively $(P<0.01$; Fig. 1). These $\mathrm{BP}$ changes were accompanied by a lowering of HR $\left(295 \pm 3\right.$ beats $\left.\mathrm{min}^{-1}, \quad P=0.099\right)$ but RespR remained unchanged. The decreased $\mathrm{BP}$ and HR values approached those of normotensive animals. At the same time, SHRs microinjected with LVV-eGFP were showing increased values of systolic $(174 \pm 10 \mathrm{mmHg} ; P>0.05)$, diastolic $(149 \pm 11 \mathrm{mmHg} ; \quad P>0.05)$ and mean $\mathrm{BP}$ $(157 \pm 10 \mathrm{mmHg} ; P>0.05)$, together with a significantly decreased HR $\left(285 \pm 6\right.$ beats $\left.\min ^{-1} ; P<0.01\right)$. This profile of BP and $\mathrm{HR}$ changes was expected and is a consequence of maturation. In contrast, no significant changes in BP, HR and RespR were observed in WKY rats during the 60 day duration of the experimental protocol.

\section{Effect of LVV-hKir2.1 microinjection on sympathetic output measured indirectly}

Spontaneously hypertensive rats showed putative evidence for an overall decrease of cardiovascular autonomic outflow at 60 days after LVV-hKir2.1 microinjection when compared with basal autonomic output at day 0 . In fact, 
by using fast Fourier transform applied to SBP and $\mathrm{R}-\mathrm{R}$ intervals, a decrease of $\mathrm{LF}_{\mathrm{SBP}} / \mathrm{HF}_{\mathrm{RR}}$ ratio (from $0.07 \pm 0.02$ to $0.04 \pm 0.01 \mathrm{mmHg}^{2} \mathrm{~ms}^{-2} ; \quad P>0.05$ ) was observed, mainly due to a strong decrease in sympathetic output expressed by $\mathrm{LF}_{\mathrm{SBP}}$ band power (from $0.79 \pm 0.13$ to $\left.0.42 \pm 0.09 \mathrm{mmHg}^{2} ; P<0.05\right)$. In SHRs, the basal $\mathrm{HF}_{\mathrm{SBP}}\left(0.75 \pm 0.10 \mathrm{mmHg}^{2}\right)$ was first reduced at 40 days and persisted until 60 days $\left(0.33 \pm 0.10 \mathrm{~mm} \mathrm{Hg}^{2}\right.$; $P<0.05)$ after LVV-hKir2.1, but it was unchanged in the LVV-eGFP group $\left(0.82 \pm 0.38 \mathrm{mmHg}^{2}\right)$. Interestingly, LF SBP was significantly reduced by 20 days after LVV-hKir2.1 microinjection and occurred before the fall in SBP. In contrast, at 60 days the $\mathrm{LF}_{\mathrm{SBP}} / \mathrm{HF}_{\mathrm{RR}}$ ratio for SHR LVV-eGFP was $0.08 \pm 0.03 \mathrm{mmHg}^{2}$ $\mathrm{ms}^{-2}$ and the LF was $0.86 \pm 0.21 \mathrm{mmHg}^{2}(P>0.05)$. The variations of mean $\mathrm{LF}_{\mathrm{SBP}}$ and $\mathrm{LF}_{\mathrm{SBP}} / \mathrm{HF}_{\mathrm{RR}}$, at 10 day intervals for each SHR group, are depicted in Fig. 2. For $\mathrm{WKY}$ rats in basal conditions, the $\mathrm{LF}_{\mathrm{SBP}}$ and $\mathrm{LF}_{\mathrm{SBP}} / \mathrm{HF}_{\mathrm{RR}}$ ratio were $3.23 \pm 0.36 \mathrm{mmHg}^{2}$ and $0.43 \pm 0.14 \mathrm{mmHg}^{2} \mathrm{~ms}^{-2}$, respectively. No significant changes in $\mathrm{LF}$ and $\mathrm{LF}_{\mathrm{SBP}} / \mathrm{HF}_{\mathrm{RR}}$ ratio were observed for WKY LVV-hKir2.1 $\left(3.11 \pm 0.44 \mathrm{mmHg}^{2}\right.$ and $0.40 \pm 0.23 \mathrm{mmHg}^{2} \mathrm{~ms}^{-2}$, respectively) and WKY LVVeGFP rats $\left(2.56 \pm 0.48 \mathrm{mmHg}^{2}\right.$ and $0.22 \pm 0.08 \mathrm{mmHg}^{2}$ $\mathrm{ms}^{-2}$, respectively).

\section{Arterial baroreflex gain and peripheral chemoreflex responsiveness}

The injection of phenylephrine triggered, in all animal groups, a progressive increase in mean BP, which was accompanied by a progressive reduction in HR. In SHRs, BRG increased significantly after LVV-hKir2.1 microinjection and approached the values of the normal control rats. The SHR LVV-hKir2.1 group had a higher BRG than the SHR LVV-eGFP group $(0.51 \pm 0.06$ versus $0.33 \pm 0.03$ beats $\mathrm{min}^{-1} \mathrm{mmHg}^{-1}$, respectively; $P<0.05$; Fig. 3). Interestingly, the BRG of WKY LVVhKir2.1 rats $\left(1.29 \pm 0.18\right.$ beats $\left.\mathrm{min}^{-1} \mathrm{mmHg}^{-1}\right)$ was also increased in comparison to the WKY LVV-eGFP group $\left(0.41 \pm 0.02\right.$ beats $\left.\mathrm{min}^{-1} \mathrm{mmHg}^{-1} ; P<0.0001\right)$, despite all cardiovascular variables remaining unchanged.

Respiratory rate remained unchanged throughout the full experimental protocol in all animal groups, before and after the lentiviral microinjection. At 60 days after microinjection, the baseline values of respiratory rate in the anaesthetized animals were $76 \pm 3.4,81 \pm 4.9,80 \pm 4.5$ and $67 \pm 3.5$ breaths $\mathrm{min}^{-1}$, respectively, for SHR and WKY LVV-hKir2.1, SHR and WKY LVV-eGFP. However, peripheral chemoreceptor reflex activation with lobeline elicited a hyperventilatory reflex response of different magnitude according to the animal group. The SHR LVV-hKir2.1 animals showed a decreased ventilatory response when compared with the SHR LVV-eGFP group $(\Delta 24.4 \pm 3.4$ versus $\Delta 38.1 \pm 4.9$ breaths $\mathrm{min}^{-1}$, respectively; $P<0.05$; Fig. 3 ). In contrast, there were no differences in the ventilatory response between WKY LVV-hKir2.1 and WKY-eGFP groups $\left(\Delta 23.3 \pm 5.9\right.$ breaths $\mathrm{min}^{-1}$ for WKY LVV-hKir2.1 and $\Delta 24.8 \pm 4.2$ breaths $\mathrm{min}^{-1}$ for WKY LVV-eGFP). Mean BP responses to chemoreflex activation in SHR LVVhKir2.1 animals (from $140 \pm 7$ to $154 \pm 9 \mathrm{mmHg}$ ) were depressed compared with SHR LVV-eGFP rats (179 \pm 9 to $193 \pm 9 \mathrm{mmHg} ; P<0.05$ ), but HR responses were not different (from $337 \pm 23$ to $359 \pm 12$ versus from $373 \pm 10$ to $362 \pm 13$ beats $\mathrm{min}^{-1}$, respectively). For the two WKY groups, changes in BP and HR in response to peripheral chemoreflex activation were not different.
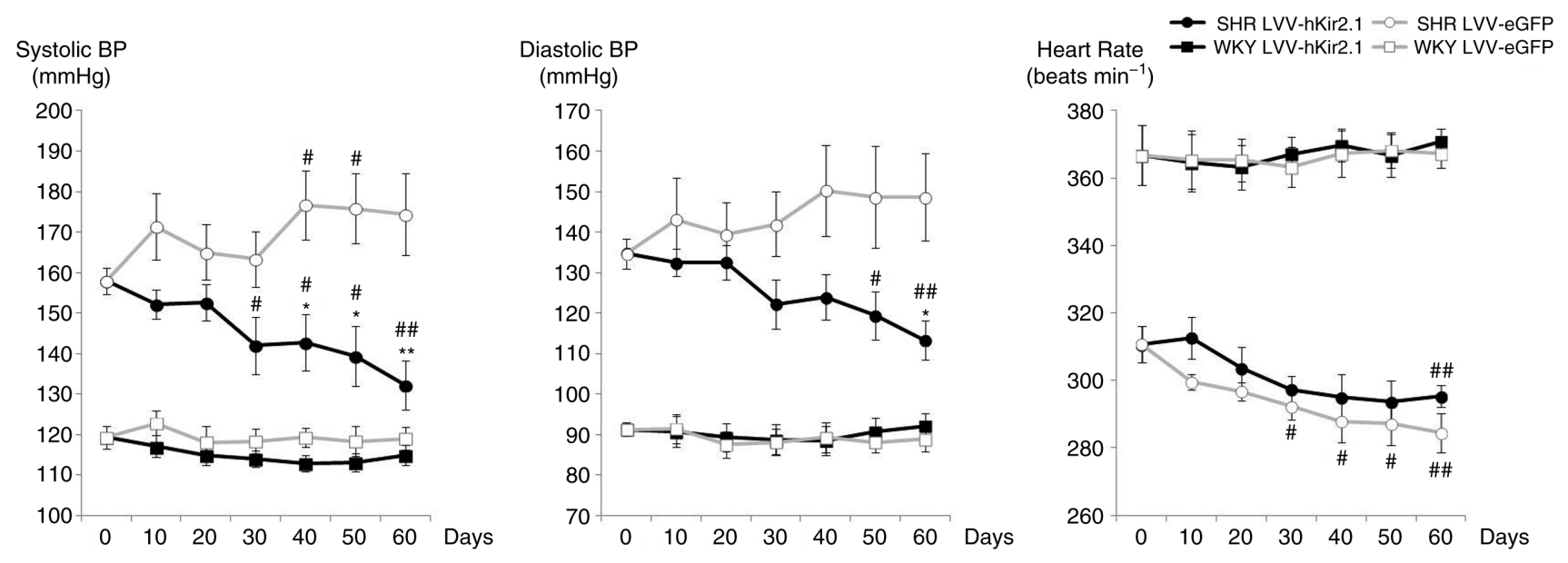

Figure 1. Effect on systolic blood pressure, diastolic blood pressure and heart rate before ( 0 days) and after microinjection of LVV-hKir2.1 $(n=7)$ or LVV-eGFP $(n=7)$

${ }^{*} P<0.05, * * P<0.01$, statistically significant differences between spontaneously hypertensive rat (SHR) LVV-hKir2.1 and SHR LVV-eGFP groups. \#P<0.05, \#\#P<0.01, statistically significant differences within the group. 


\section{Circadian variation of BP and HR and patterns of nocturnal blood pressure profile}

In basal conditions and without any intervention, the pattern of circadian variation of BP and HR followed a similar trend, with lower BP values during the light phase relative to the dark phase. During the light phase, the systolic, diastolic and mean BP of SHRs were significantly higher than those for WKY rats (Table 1; $P<0.0001$ ) over the same time period. The same type of variation was found for the dark phase, during which SHRs showed higher values for BP parameters than WKY rats $(P<0.0001$; Table 1). Mean basal HR followed these variations in $\mathrm{BP}$ inversely. The HR was significantly lower during the light and dark phases for SHRs than for WKY rats $(P<0.01$; Table 1$)$.

At 60 days after the LVV-hKir2.1 microinjection, SHRs showed a significant decrease of systolic, diastolic and mean BP during both the light and the dark phase (both $P<0.01$; Table 1). A significant decrease of HR was observed during the light but not during the dark phase $(P>0.05)$. For the SHR LVV-eGFP rats, HR, diastolic, systolic and mean BP values for the light phase and dark phase were increased as expected at 60 days (Table 1). Finally, in WKY LVV-hKir2.1 as well as WKY LVV-eGFP rats there was an increase in BP during the dark phase without a distinct circadian rhythm. This profile was maintained at 60 days after LVV-hKir2.1 and LVV-eGFP PVN microinjections (Table 1).

\section{Metabolic evaluation}

A significant decrease in food intake was observed in the SHR LVV-hKir2.1 group at 60 days after the microinjection (Table 2). No other significant changes were found in body weight, water intake, faeces and urine production for all groups, before and after the microinjections, suggesting that the physical inactivity due to social isolation (only one animal per cage) could have an impact on food consumption. Furthermore, animals were not subjected to an adaptation period to the metabolic cages, which could impact on our metabolic data, constituting a study limitation.

\section{Histological, immunohistochemical and Western blot analysis}

The microinjection sites were located within the PVN according to the rat atlas of Paxinos \& Watson (1986). Enhanced green fluorescent protein was detected by fluorescence microscopy as fluorescence confined to a surface of $0.10-0.20 \mathrm{~mm}$ around the injection site. The eGFP did not penetrate the third ventricular ependymal lining. Through immunohistochemical studies, it was confirmed that PVN neurones expressed eGFP (Fig. 4).

The overexpression of hKir2.1 in the PVN was analysed using Western blot. The PVN dissected from SHRs microinjected with LVV-hKir2.1 showed an increased expression of hKir2.1, on average about ninefold increased when compared with the LVV-eGFP group (Fig. 4).

\section{Discussion}

In the present study, we investigated the effect of overexpressing an inwardly rectifying potassium channel

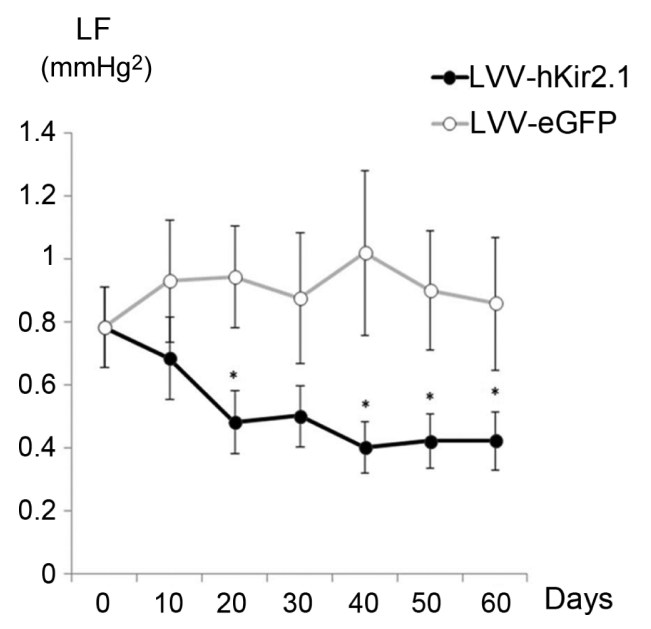

LF[BP]/ HF[RR]

$\left(\mathrm{mmHg}^{2} \mathrm{~ms}^{-2}\right)$

$\rightarrow-L V V-h K i r 2.1$

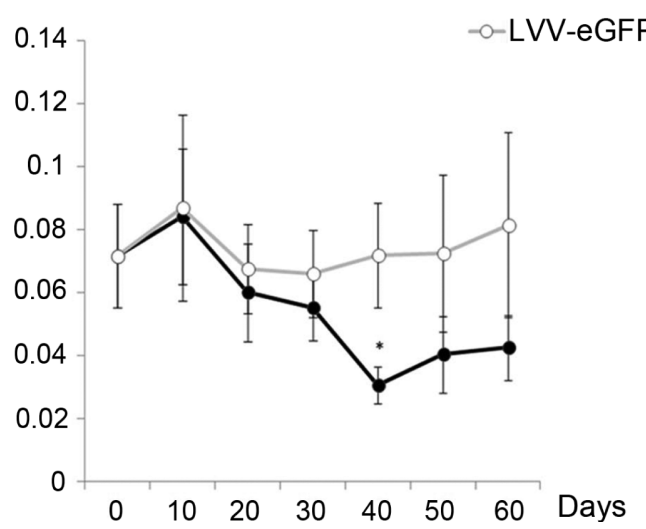

Figure 2. Mean ( \pm SEM) low frequency (LF) and ratio of low (blood pressure) to high frequency (R-R interval) [LF(BP)/HF(RR)] before (0 days) and at 10 day intervals after the microinjection of LVV-hKir2.1 or LVV-eGFP in SHRs

Note that the fall in LF systolic blood pressure (SBP) occurred a week before the fall in SBP, suggesting a causative association. ${ }^{*} P<0.05$, statistically significant difference between groups. 
in the PVN to lower neuronal activity, while measuring BP chronically, as well as its reflex control, in a rat model of hypertension. Our study is the first to demonstrate that chronic suppression of PVN neuronal activity in freely moving SHRs causes a sustained reduction in arterial blood pressure ( $>60$ days) together with a decrease of sympathetic activity, a downregulation of peripheral chemoreflex responsiveness and an improvement of baroreflex gain. No such changes were found in the control groups of both rat strains that underwent comparable experimental protocols.

The PVN of the hypothalamus is well known for its importance in autonomic control and, in particular, for cardiovascular regulation. Several anatomical and electrophysiological studies have shown that PVN neurones project either directly to the spinal cord or to the rostral ventrolateral medulla (Coote, 2007), thereby accessing sympathetic neurones to modulate blood pressure (Hosoya et al. 1991; Loewy, 1991; Coote, 1995, 2005; Ranson et al. 1998; Motawei et al. 1999; Pyner \& Coote, 1999, 2000; Badoer, 2001). As an example, electrolytic lesions of the PVN in SHRs elicited an acute reduction of sympathetic activity together with a decrease of blood pressure (Takeda et al. 1991). Other acute studies, performed under general anaesthesia, showed that muscimol injections into the PVN lowered BP and renal sympathetic nerve activity in both SHRs and WKY rats, indicating that this region was tonically active in both animal strains to control BP and peripheral sympathetic activity (Allen, 2002). In the SHR, sympathetic activity is known to be overactivated even before hypertension develops (Simms et al. 2009). Several studies have pointed out that the persistent increase in sympathetic tone is a major contributor to both the initiation and the maintenance of the hypertensive condition (Yamada et al. 1988; Grassi, 2004b; Smith et al. 2004; Guyenet, 2006; Fisher \& Paton, 2012). In fact, increased sympathetic activity has been detected in normotensive individuals with a family history of hypertension and in individuals with essential hypertension, but not in those with secondary hypertension (Yamada et al. 1988; Grassi et al. 1998; Grassi, 2004a, 2009). Likewise, high plasmatic noradrenaline levels have also been associated with essential hypertension, being consistently increased in younger hypertensive patients (Grassi, 1998), and increased peripheral sympathetic nervous activity has been detected by microneurographic techniques in hypertensive patients (Anderson et al. 1989; Grassi, 1998; Greenwood et al. 1999; Mano, 2012). Several studies, both in human subjects and in animal models, have demonstrated an association between the circadian variation of BP values, the hypertensive condition, the sympathetic activation, the end-organ damage and the worsening of cardiovascular outcome (White, 2000; Pickering \& Kario, 2001; Weber, 2002). Thus, the idea of a long-term modulation of the level of sympathetic activity, at its central origin, as a way to control and treat high blood pressure and to increase cardiovascular compliance, is very appealing. In particular, the manipulation of sympathetic cell excitability by modulation of $\mathrm{K}^{+}$channel expression, to hyperpolarize neuronal resting membrane potential, is an attractive hypothetical therapeutic strategy (Duale et al. 2007).

In the present work, our purpose was to depress the activity of PVN neurones chronically by the overexpression of $\mathrm{K}^{+}$channels exclusively in PVN
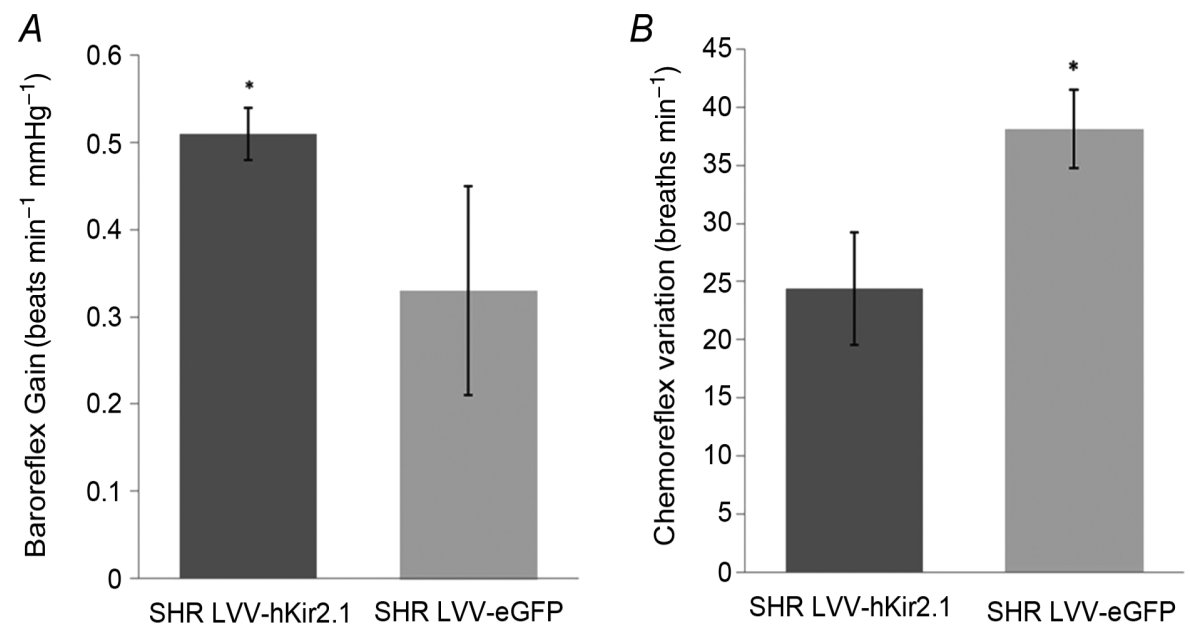

\footnotetext{
Figure 3. Effect of bilateral microinjections of LVV-hKir2.1 or LVV-eGFP into the paraventricular nucleus on baroreflex gain $(A)$ and chemoreflex variation $(B), 60$ days after microinjection In the SHR LVV-hKir2.1 group, there is an increase in the baroreflex gain and a decrease in the chemoreflex ventilatory response. ${ }^{*} P<0.05$, statistically significant differences between groups.
} 
Table 1. Blood pressure (in millimetres of mercury) and heart rate (in beats per minute) during the light and dark phases for all groups before and 59 days after the microinjection

\begin{tabular}{|c|c|c|c|c|c|c|c|c|}
\hline \multirow[b]{2}{*}{ Group } & \multicolumn{4}{|c|}{ Light phase } & \multicolumn{4}{|c|}{ Dark phase } \\
\hline & SBP & DBP & MBP & $\mathrm{HR}$ & SBP & DBP & MBP & $\mathrm{HR}$ \\
\hline \multicolumn{9}{|l|}{ Basal } \\
\hline SHR & $156 \pm 3$ & $132 \pm 3$ & $140 \pm 3$ & $297 \pm 6$ & $160 \pm 4$ & $137 \pm 4$ & $145 \pm 4$ & $325 \pm 6$ \\
\hline WKY & $118 \pm 3$ & $90 \pm 2$ & $100 \pm 1$ & $362 \pm 9$ & $120 \pm 3$ & $92 \pm 2$ & $102 \pm 2$ & $373 \pm 11$ \\
\hline \multicolumn{9}{|c|}{59 days after microinjection } \\
\hline SHR LVV-hKir2.1 & $131 \pm 5^{*}$ & $113 \pm 4^{*}$ & $119 \pm 4^{*}$ & $271 \pm 2$ & $133 \pm 7^{*}$ & $114 \pm 6^{*}$ & $120 \pm 6^{*}$ & $320 \pm 5^{*}$ \\
\hline SHR LVV-eGFP & $172 \pm 11$ & $145 \pm 11$ & $154 \pm 10$ & $264 \pm 5$ & $177 \pm 10$ & $152 \pm 11$ & $160 \pm 10$ & $305 \pm 7$ \\
\hline WKY LVV-hKir2.1 & $117 \pm 4$ & $87 \pm 3$ & $97 \pm 2$ & $340 \pm 10$ & $121 \pm 2$ & $91 \pm 4$ & $101 \pm 3$ & $378 \pm 12$ \\
\hline WKY LVV-eGFP & $114 \pm 2$ & $88 \pm 3$ & $96 \pm 2$ & $354 \pm 5$ & $116 \pm 2$ & $92 \pm 3$ & $100 \pm 2$ & $389 \pm 2$ \\
\hline
\end{tabular}

Values are expressed as means \pm SEM. Abbreviations: DBP, diastolic blood pressure; HR, heart rate; MBP, mean blood pressure; and SBP, systolic blood pressure; SHR LVV-hKir2.1, Spontaneously hypertensive rats microinjected with LVV-hKir2.1; SHR LVV-eGFP, Spontaneously hypertensive rats microinjected with LVV-eGFP; WKY LVV-hKir2.1, Wistar Kyoto rats microinjected with LVV-hKir2.1; WKY LVV-eGFP, Wistar Kyoto rats microinjected with LVV-eGFP. ${ }^{*} P<0.01$, statistically significant difference between basal and day 59 values.

Table 2. Metabolic evaluation of spontaneously hypertensive rats before injection and 59 days afterwards

\begin{tabular}{|c|c|c|c|c|c|}
\hline Group & $\Delta$ Weight (g) & Food (g) & Water $(\mathrm{ml})$ & Faeces $(g)$ & Urine $(\mathrm{ml})$ \\
\hline \multicolumn{6}{|c|}{ Before microinjection; basal conditions } \\
\hline SHR LVV-eGFP & $-1 \pm 2.0$ & $19 \pm 3.5$ & $27 \pm 2.2$ & $9 \pm 2.1$ & $11 \pm 1$ \\
\hline SHR LVV-hkir2.1 & $-1 \pm 1.4$ & $24 \pm 1.3$ & $40 \pm 4.2$ & $14 \pm 3.1$ & $16 \pm 3.5$ \\
\hline \multicolumn{6}{|c|}{ After microinjection (at 59 days) } \\
\hline SHR LVV-eGFP & $-3 \pm 1.3$ & $27 \pm 1.6$ & $31 \pm 4.0$ & $13 \pm 1.5$ & $12 \pm 0.9$ \\
\hline SHR LVV-hkir2.1 & $-1 \pm 0.6$ & $20 \pm 0.6^{*}$ & $32 \pm 4.9$ & $8 \pm 0.9$ & $16 \pm 2.1$ \\
\hline
\end{tabular}

Values are expressed as means \pm SEM. Abbreviations: SHR LVV-hKir2.1, Spontaneously hypertensive rats microinjected with LVV-hKir2.1; SHR LVV-eGFP, Spontaneously hypertensive rats microinjected with LVV-eGFP; WKY LVV-hKir2.1, Wistar Kyoto rats microinjected with LVV-hKir2.1; WKY LVV-eGFP, Wistar Kyoto rats microinjected with LVV-eGFP. ${ }^{*} P<0.05$, statistically significant difference between basal and day 59 values.
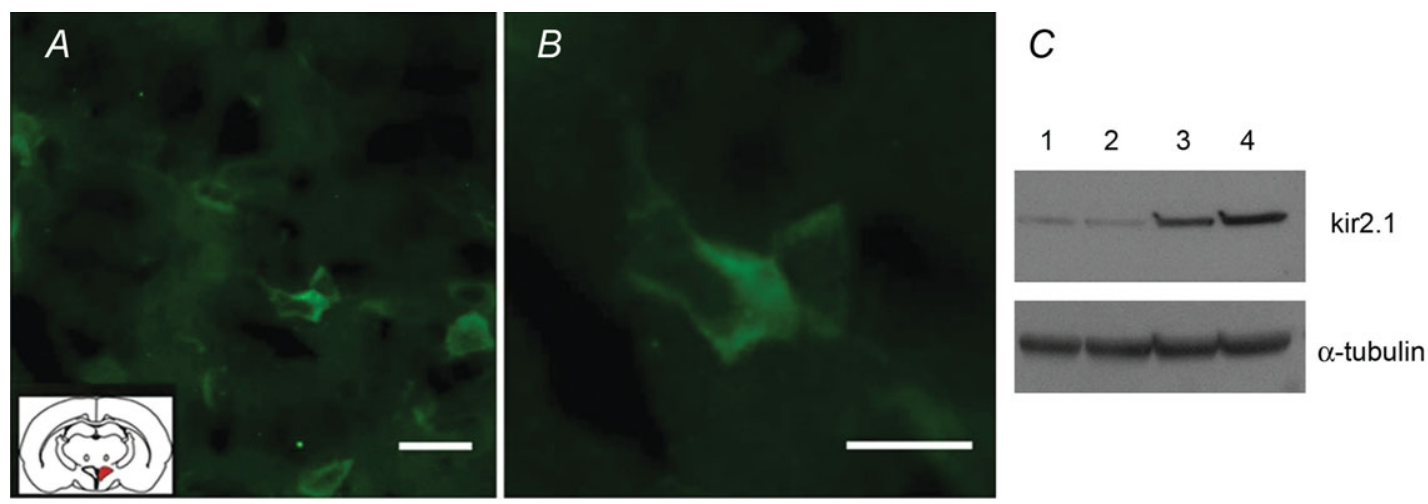

Figure 4. Lentiviral vector-mediated transduction of enhanced green fluorescent protein (eGFP) in the paraventricular nucleus

Confocal microscope images of eGFP-expressing cells in the paraventricular nucleus following injection of lentiviral vector into this site. Scale bar in $A$ represents $20 \mu \mathrm{m}$ and scale bar in $B$ represents $10 \mu \mathrm{m}$. C, Western blot analysis of sham-treated SHRs (lanes 1 and 2) and LVV-hKir2.1 microinjected SHRs (lanes 3 and 4). Results show an overexpression of hKir2.1 in LVV-hKir2.1-microinjected SHRs. $\alpha$-Tubulin was used as the housekeeping gene. 
neurones, in order to evaluate its consequences upon long-term blood pressure regulation in an animal model of hypertension. We overexpressed a human inwardly rectifying potassium channel (hKir2.1) under the control of a synapsin promoter that was neurone specific (Duale et al. 2005a; Duale et al. 2005b). Lentivirus was used because its expression has been shown to be sustained within PVN neurones in the long term (Coleman et al. 2003). In previous studies, Duale et al. (2007) and Howorth et al. (2009) showed that hKir2.1 overexpression hyperpolarized the membrane potential of cultured cathecolaminergic PC12 cells by $\sim 10 \mathrm{mV}$, which is expected to result in 'electrical silencing' of PVN neurones (Duale et al. 2007; Howorth et al. 2009). Similar overexpression strategies have been used to reveal that electrical silencing of neurones affected development in ovo (Yoon et al. 2008), neuronal activity in vivo (Okada \& Matsuda, 2008) and the ability of neurones to make and maintain connections in vivo (Yu et al. 2004; Mizuno et al. 2007; Hendy, 2010). This virusmediated approach has the advantage of being site specific and enabling overexpression in adulthood, which avoids the development of putative compensatory mechanisms associated with transgenic animals (Hendy, 2010).

Our results show that LVV-hKir2.1 treatment of the PVN in SHRs lowered SBP by $\sim 15 \%(>20 \mathrm{mmHg})$. This decline of SBP, which was accompanied by a decrease in HR, was statistically confirmed at 30 days after the lentiviral microinjection and persisted until the animals were killed 60 days postinjection. Interestingly, the LF spectra of SBP (indicative of sympathoinhibition) occurred before the fall in SBP (i.e. 20 versus 30 days), suggesting a putative association between the changes in both variables. Furthermore, the fall in HF SBP is indicative of reduced respiratory modulation of arterial pressure and could include reduced respiratorysympathetic coupling, a phenomenon known to raise total peripheral resistance in the SHR (Simms et al. 2009). In contrast, changes in diastolic BP were significant only after 50 days, suggesting the involvement of an additional mechanism. This reveals novel insight into the long-term control of arterial pressure in hypertension by the PVN. It also indicates that the system does not adapt. This could be explained by the associated improvement of baroreflex gain and/or a downregulation of peripheral chemoreflex responsiveness to stabilize lower levels of blood pressure, as we observed. We propose that these changes were a result of reduced electrical excitability of PVN premotor sympathetic neurones, but we cannot rule out reduced release of vasopressin and oxytocin. This is consistent with our neuroanatomical Western blot analysis confirming that hKir 2.1 protein overexpression was within the PVN region. Interestingly, respiratory rate remained unchanged in all experimental groups, suggesting that there is no tonic excitatory drive from the PVN affecting this variable in hypertensive or normotensive rats. Additionally, we saw no tonic influence from the PVN on the resting arterial pressure level in normotensive rats, which contrasts with a previous acute in vivo study (Allen, 2002).

It is well accepted that neurogenic hypertension is accompanied by an impairment of the baroreceptor reflex (Grassi et al. 1998). Our data showed that depressing PVN neuronal activity improved baroreflex gain. Previous work from several authors has shown that during the course of an alerting reaction there is a decrease in baroreflex efficacy and a facilitation of the carotid chemoreceptor reflex due to modifications of synaptic integration at the level of the nucleus tractus solitarii; this might include mechanisms involving GABA and angiotensin II release within the nucleus tractus solitarii (Jordan et al. 1988; Spyer K, 1990; Silva-Carvalho et al. 1995a, b; Kasparov et al. 1998; Kasparov \& Paton, 1999; Head \& Mayorov, 2001; Rocha et al. 2003). Such an angiotensinogenic mechanism seems to be particularly active in pathophysiological conditions such as myocardial ischaemia and hypertension (Rocha et al. 2003; Rosário et al. 2003; Maximino et al. 2006), and its behaviour can be modulated by intervening pharmacologically on $\mathrm{AT}_{1}$ receptors within the nucleus tractus solitarii (Kasparov et al. 1998; Kasparov \& Paton, 1999; Rocha et al. 2003; Rosário et al. 2003). In fact, during myocardial ischaemia, $\mathrm{AT}_{1}$ blockade reversed the remodelling of baroreceptor and chemoreceptor reflex function in a way similar to that elicited upon the overexpression of hKir2.1 in PVN neuronal cells (Rocha et al. 2003; Rosário et al. 2003).

The demonstration of a non-dipper blood pressure profile in animal models remains difficult, mainly due to the failure to establish a clear distinction between day and night values. This was confirmed in our study, because through $\mathrm{PVN}$-induced sympathetic manipulations, we were only able to modify BP light-dark values of SHRs which approached those of WKY rats. However, we were unable to modify the day and night profile of BP value variations in both strains. This inability to define a lightdark profile in rats similar to the one set for human subjects may be due to the intermittent behaviour rats, with alternating awake and sleep periods in both the light and the dark phase. It is likely that the only way to define the light and dark phase profiles of rats better would be by monitoring of cerebral activity through EEG, which was outside the scope of the present work.

In conclusion, the present work shows that the intervention on central sympathoexcitatory neurone excitability through the genetic manipulation of expression of $\mathrm{K}^{+}$channels is able to alter peripheral blood pressure in the long term. This occurs by remodelling of the sympathetic outflow and restores the imbalance of peripheral reflex mechanisms that maintain cardiovascular homeostasis. Our data, from an 
animal model, give insights into the pathophysiological mechanisms involved in the aetiology of neurogenic hypertension and provide novel hypothetical therapeutic interventions at both the central and the peripheral level of the autonomic nervous system to control sympatoexcitation.

\section{References}

Allen AM (2002). Inhibition of the hypothalamic paraventricular nucleus in spontaneously hypertensive rats dramatically reduces sympathetic vasomotor tone. Hypertension 39, 275-280.

Anderson EA, Sinkey CA, Lawton WJ \& Mark AL (1989). Elevated sympathetic nerve activity in borderline hypertensive humans. Evidence from direct intraneural recordings. Hypertension 14, 177-183.

Badoer E (2001). Hypothalamic paraventricular nucleus and cardiovascular regulation. Clin Exp Pharmacol Physiol 28, 95-99.

Caverson MM, Ciriello J \& Calaresu FR (1984). Paraventricular nucleus of the hypothalamus: an electrophysiological investigation of neurons projecting directly to intermediolateral nucleus in the cat. Brain Res 305, 380-383.

Cerutti C, Gustin MP, Paultre CZ, Lo M, Julien C, Vincent M \& Sassard J (1991). Autonomic nervous system and cardiovascular variability in rats: a spectral analysis approach. Am J Physiol Heart Circ Physiol 261, H1292-H1299.

Ciriello J, Kline RL, Zhang TX \& Caverson MM (1984). Lesions of the paraventricular nucleus alter the development of spontaneous hypertension in the rat. Brain Res 310, 355-359.

Coleman JE, Huentelman MJ, Kasparov S, Metcalfe BL, Paton JF, Katovich MJ, Semple-Rowland SL \& Raizada MK (2003). Efficient large-scale production and concentration of HIV-1-based lentiviral vectors for use in vivo. Physiol Genomics 12, 221-228.

Coote JH (1995). Cardiovascular function of the paraventricular nucleus of the hypothalamus. Biol Signals 4, $142-149$.

Coote JH (2005). A role for the paraventricular nucleus of the hypothalamus in the autonomic control of heart and kidney. Exp Physiol 90, 169-173.

Coote JH (2007). Landmarks in understanding the central nervous control of the cardiovascular system. Exp Physiol 92, 3-18.

Dampney RA, Horiuchi J, Killinger S, Sheriff MJ, Tan PS \& McDowall LM (2005). Long-term regulation of arterial blood pressure by hypothalamic nuclei: some critical questions. Clin Exp Pharmacol Physiol 32, 419-425.

Duale H, Kasparov S, Paton JF \& Teschemacher AG (2005a). Differences in transductional tropism of adenoviral and lentiviral vectors in the rat brainstem. Exp Physiol 90, 71-78.

Duale H, Kasparov S, Paton J, Teschemacher A \& Waki H $(2005 b)$. The role of A2 NAergic neurones in cardiovascular regulation. J Physiol, 567P, PC39.

Duale H, Waki H, Howorth P, Kasparov S, Teschemacher AG \& Paton JF (2007). Restraining influence of A2 neurons in chronic control of arterial pressure in spontaneously hypertensive rats. Cardiovasc Res 76, 184-193.
Fisher JP \& Paton JF (2012). The sympathetic nervous system and blood pressure in humans: implications for hypertension. J Hum Hypertens 26, 463-475.

Furlan R, Porta A, Costa F, Tank J, Baker L, Schiavi R, Robertson D, Malliani A \& Mosqueda-Garcia R (2000). Oscillatory patterns in sympathetic neural discharge and cardiovascular variables during orthostatic stimulus. Circulation 101, 886-892.

Goto A, Ikeda T, Tobian L, Iwai J \& Johnson MA (1981). Brain lesions in the paraventricular nuclei and catecholaminergic neurons minimize salt hypertension in Dahl salt-sensitive rats. Clin Sci (Lond) 61 Suppl 7, 53s-55s.

Grassi G (1998). Role of the sympathetic nervous system in human hypertension. J Hypertens 16, 1979-1987.

Grassi G (2004a). Counteracting the sympathetic nervous system in essential hypertension. Curr Opin Nephrol Hypertens 13, 513-519.

Grassi G (2004b). Sympathetic and baroreflex function in hypertension: implications for current and new drugs. Curr Pharm Des 10, 3579-3589.

Grassi G (2009). Assessment of sympathetic cardiovascular drive in human hypertension: achievements and perspectives. Hypertension 54, 690-697.

Grassi G, Cattaneo BM, Seravalle G, Lanfranchi A \& Mancia G (1998). Baroreflex control of sympathetic nerve activity in essential and secondary hypertension. Hypertension 31, 68-72.

Greenwood JP, Stoker JB \& Mary DASG (1999). Single-unit sympathetic discharge: quantitative assessment in human hypertensive disease. Circulation 100, 1305-1310.

Guyenet PG (2006). The sympathetic control of blood pressure. Nat Rev Neurosci 7, 335-346.

Hardy SG (2001). Hypothalamic projections to cardiovascular centers of the medulla. Brain Res 894, 233-240.

Head GA \& Mayorov DN (2001). Central angiotensin and baroreceptor control of circulation. Ann N Y Acad Sci 940, 361-379.

Hendy E (2010). Sites and mechanisms within the brainstem for chronic regulation of arterial blood pressure, $\mathrm{PhD}$ Thesis. University of Bristol.

Herzig TC, Buchholz RA \& Haywood JR (1991). Effects of paraventricular nucleus lesions on chronic renal hypertension. Am J Physiol Heart Circ Physiol 261, H860-H867.

Hosoya Y, Sugiura Y, Okado N, Loewy AD \& Kohno K (1991). Descending input from the hypothalamic paraventricular nucleus to sympathetic preganglionic neurons in the rat. Exp Brain Res 85, 10-20.

Howorth PW, Thornton SR, O'Brien V, Smith WD, Nikiforova N, Teschemacher AG \& Pickering AE (2009). Retrograde viral vector-mediated inhibition of pontospinal noradrenergic neurons causes hyperalgesia in rats. J Neurosci 29, 12855-12864.

Japundzic N, Grichois ML, Zitoun P, Laude D \& Elghozi JL (1990). Spectral analysis of blood pressure and heart rate in conscious rats: effects of autonomic blockers. J Auton Nerv Syst 30, 91-100.

Jordan D, Mifflin SW \& Spyer KM (1988). Hypothalamic inhibition of neurones in the nucleus tractus solitarius of the cat is GABA mediated. J Physiol 399, 389-404. 
Kannan H, Hayashida Y \& Yamashita H (1989). Increase in sympathetic outflow by paraventricular nucleus stimulation in awake rats. Am J Physiol Regul Integr Comp Physiol 256, R1325-R1330.

Kasparov S, Butcher JW \& Paton JF (1998). Angiotensin II receptors within the nucleus of the solitary tract mediate the developmental attenuation of the baroreceptor vagal reflex in pre-weaned rats. J Auton Nerv Syst 74, 160-168.

Kasparov S \& Paton JFR (1999). Differential effects of angiotensin II in the nucleus tractus solitarii of the rat plausible neuronal mechanism. J Physiol 521, 227-238.

Loewy AD (1991). Forebrain nuclei involved in autonomic control. Prog Brain Res 87, 253-268.

Malliani A, Pagani M, Lombardi F \& Cerutti S (1991). Cardiovascular neural regulation explored in the frequency domain. Circulation 84, 482-492.

Mano T (2012). Clinical applications of microneurography. In Primer on the Autonomic Nervous System, Edited by: David Robertson, Italo Biaggioni, Geoffrey Burnstock, Phillip A. Low and Julian F.R. Paton, pp. 393-397. Academic Press, London, UK/San Diego, CA, USA, Waltham, MA, USA.

Marques-Neves C, Martins-Baptista A, Boto JP, Delgado E, Silva-Carvalho L \& Rocha I (2004). Intraocular pressure variability in the anesthetized rat: a spectral analysis. Eur J Ophthalmol 14, 381-386.

Maximino JR, Ferrari MF, Coelho EF \& Fior-Chadi DR (2006). Time course analysis of tyrosine hydroxylase and angiotensinogen mRNA expression in central nervous system of rats submitted to experimental hypertension. Neurosci Res 55, 292-299.

Mizuno H, Hirano T \& Tagawa Y (2007). Evidence for activity-dependent cortical wiring: formation of interhemispheric connections in neonatal mouse visual cortex requires projection neuron activity. J Neurosci 27 , 6760-6770.

Motawei K, Pyner S, Ranson RN, Kamel M \& Coote JH (1999). Terminals of paraventricular spinal neurones are closely associated with adrenal medullary sympathetic preganglionic neurones: immunocytochemical evidence for vasopressin as a possible neurotransmitter in this pathway. Exp Brain Res 126, 68-76.

Okada M \& Matsuda H (2008). Chronic lentiviral expression of inwardly rectifying $\mathrm{K}^{+}$channels (Kir2.1) reduces neuronal activity and downregulates voltage-gated potassium currents in hippocampus. Neuroscience 156, 289-297.

Paxinos G \& Watson C(1986). The Rat Brain in Stereotaxic Coordinates. 2nd edition. Academic Press, London, UK.

Pickering TG (2003). Isolated diastolic hypertension. J Clin Hypertens (Greenwich) 5, 411-413.

Pickering TG \& Kario K (2001). Nocturnal non-dipping: what does it augur? Curr Opin Nephrol Hypertens 10, 611-616.

Pyner S \& Coote JH (1999). Identification of an efferent projection from the paraventricular nucleus of the hypothalamus terminating close to spinally projecting rostral ventrolateral medullary neurons. Neuroscience $\mathbf{8 8}$, 949-957.

Pyner S \& Coote JH (2000). Identification of branching paraventricular neurons of the hypothalamus that project to the rostroventrolateral medulla and spinal cord. Neuroscience 100, 549-556.
Radaelli A, Bernardi L, Valle F, Leuzzi S, Salvucci F, Pedrotti L, Marchesi E, Finardi G \& Sleight P (1994). Cardiovascular autonomic modulation in essential hypertension. Effect of tilting. Hypertension 24, 556-563.

Ranson RN, Motawei K, Pyner S \& Coote JH (1998). The paraventricular nucleus of the hypothalamus sends efferents to the spinal cord of the rat that closely appose sympathetic preganglionic neurones projecting to the stellate ganglion. Exp Brain Res 120, 164-172.

Rocha I, Brás-Rosário L, Amparo-Barros M \& Silva-Carvalho L (2003). Angiotensin AT1 receptor antagonist losartan and the defence reaction in the anaesthetised rat. Effect on the carotid chemoreflex. Exp Physiol 88, 309-314.

Rosário LB, Rocha I \& Silva-Carvalho L (2003). Effect of losartan microinjections into the NTS on the cardiovascular components of chemically evoked reflexes in a rabbit model of acute heart ischemia. Adv Exp Med Biol 536, 423-431.

Schlaich MP, Hering D, Sobotka P, Krum H, Lambert GW, Lambert E \& Esler MD (2012). Effects of renal denervation on sympathetic activation, blood pressure, and glucose metabolism in patients with resistant hypertension. Front Physiol 3, 10.

Shafton AD, Ryan A \& Badoer E (1998). Neurons in the hypothalamic paraventricular nucleus send collaterals to the spinal cord and to the rostral ventrolateral medulla in the rat. Brain Res 801, 239-243.

Silva-Carvalho L, Dawid-Milner MS, Goldsmith GE \& Spyer KM (1995a). Hypothalamic modulation of the arterial chemoreceptor reflex in the anaesthetized cat: role of the nucleus tractus solitarii. J Physiol 487, 751-760.

Silva-Carvalho L, Dawid-Milner MS \& Spyer KM (1995b). The pattern of excitatory inputs to the nucleus tractus solitarii evoked on stimulation in the hypothalamic defence area in the cat. J Physiol 487, 727-737.

Simms AE, Paton JFR, Pickering AE \& Allen AM (2009). Amplified respiratory-sympathetic coupling in the spontaneously hypertensive rat: does it contribute to hypertension? J Physiol 587, 597-610.

Smith PA, Graham LN, Mackintosh AF, Stoker JB \& Mary DA (2004). Relationship between central sympathetic activity and stages of human hypertension. Am J Hypertens 17, 217-222.

Spyer K (1990). The central nervous organization of reflex circulatory control. In Central Regulation of Autonomic Functions, pp. 168-188. Oxford University Press, New York, NY/Oxford, UK.

Takeda K, Nakata T, Takesako T, Itoh H, Hirata M, Kawasaki S, Hayashi J, Oguro M, Sasaki S \& Nakagawa M (1991). Sympathetic inhibition and attenuation of spontaneous hypertension by PVN lesions in rats. Brain Res 543, 296-300.

Task Force of the European Society of Cardiology and the North American Society of Pacing and Electrophysiology (1996). Heart rate variability. Standards of measurement, physiological interpretation, and clinical use. Eur Heart $J$ 17, 354-381. 
Tavares C, Carneiro, RM, Laranjo S \& Rocha I (2011). Computational tools for assessing cardiovascular variability. In Book of 1st Portuguese Meeting in Bioengineering, 1-6. Bioengineering (ENBENG), Lisbon, Portugal.

Waki H, Kasparov S, Wong LF, Murphy D, Shimizu T \& Paton JFR (2003). Chronic inhibition of endothelial nitric oxide synthase activity in nucleus tractus solitarii enhances baroreceptor reflex in conscious rats. J Physiol 546, 233-242.

Weber MA (2002). The 24-hour blood pressure pattern: does it have implications for morbidity and mortality? Am J Cardiol 89, 27A-33A.

White WB (2000). Ambulatory blood pressure monitoring: dippers compared with non-dippers. Blood Press Monit 5 Suppl 1, S17-S23.

Yamada Y, Miyajima E, Tochikubo O, Matsukawa T, Shionoiri H, Ishii M \& Kaneko Y (1988). Impaired baroreflex changes in muscle sympathetic nerve activity in adolescents who have a family history of essential hypertension. J Hypertens Suppl 6, S525-S528.

Yamori Y \& Okamoto K (1969). Hypothalamic tonic regulation of blood pressure in spontaneously hypertensive rats. Jpn Circ J 33, 509-519.

Yoon YJ, Kominami H, Trimarchi T \& Martin-Caraballo M (2008). Inhibition of electrical activity by retroviral infection with Kir2.1 transgenes disrupts electrical differentiation of motoneurons. PLoS One 3, e2971.
Yu CR, Power J, Barnea G, O’Donnell S, Brown HE, Osborne J, Axel R \& Gogos JA (2004). Spontaneous neural activity is required for the establishment and maintenance of the olfactory sensory map. Neuron 42, 553-566.

Zhang K, Li YF \& Patel KP (2002). Reduced endogenous GABA-mediated inhibition in the PVN on renal nerve discharge in rats with heart failure. Am J Physiol Regul Integr Comp Physiol 282, R1006-R1015.

Zubcevic J, Waki H, Raizada MK \& Paton JFR (2011). Autonomic-immune-vascular interaction: an emerging concept for neurogenic hypertension. Hypertension 57, 1026-1033.

\section{Additional Information}

\section{Competing interests}

None declared.

\section{Funding}

This work was suported by Fundação para a Ciência e Tecnologia -PTDC/SAU-OSM/109081/2008, British Heart Foundation and NIH. 